\title{
Multiple brain metastases from primary gall bladder carcinoma treated by sequential surgery, radiotherapy and chemotherapy
}

\author{
Nirdosh Kumar Pant ${ }^{2 *}$, Abhishek Singh ${ }^{1 *}$ and Deepak Kumar ${ }^{3 *}$
}

\begin{abstract}
Gallbladder carcinoma tends to be an aggressive tumour that spreads early and leads to rapid death after diagnosis as compared to other gastrointestinal carcinomas. Brain metastasis in such patients further cuts short their life. The common metastasis sites from carcinoma of the gallbladder are liver, regional lymphnodes; and peritoneum. The only common extra-abdominal sites of metastasis are lungs and at few occasions skeletal system. Systemic metastases from gallbladder carcinoma occur frequently, but involvement of the central nervous system is a rarity. Surgery has shown to be a viable approach in solitary brain metastasis but multiple brain metastases are difficult to treat. In patients with multiple metastases, surgery, radiation therapy or chemotherapy alone or in combination are the options available. The treatment plan in patients with multiple metastases needs to be individualized keeping in mind various tumor and patient factors. Such individualized treatment can provide palliation to patients with improvement of quality of life; without adding to treatment related complications. Here, we present a case of carcinoma of the gallbladder with two foci of brain metastases managed with combination treatment of surgery, chemotherapy and radiotherapy. She tolerated whole treatment well without any complications. Thirteen months after her treatment, she is currently asymptomatic and is on regular follow-up.
\end{abstract}

\begin{abstract}
Introduction
Primary gallbladder carcinoma is the most common malignancy of the biliary tract and the fifth most common carcinoma of gastro-intestinal tract. Gallbladder carcinoma tends to be an aggressive tumour that spreads early and leads to rapid death after diagnosis as compared to other gastro-intestinal carcinomas. Carcinoma of the gallbladder spreads early by lymphatic metastasis, haematogenous metastasis and direct invasion into the liver. It also has a uniquely high tendency to seed the peritoneal surfaces by spillage and can cause tumour implants in biopsy tracts, surgical wounds and the peritoneal cavity. The common metastasis sites from carcinoma of the gallbladder are liver, regional lymphnodes; and peritoneum. The only common extraabdominal sites of metastasis are lungs and at few occasions skeletal system [1,2] Gallbladder carcinoma was found to be the primary site in less than $0.5 \%$ of all metastases to brain in brain metastasis studies [2-8]. Here, we present a case of carcinoma of the gallbladder with two foci of brain metastases managed with combination treatment of surgery, chemotherapy and radiotherapy. Earlier cases reported in literature had single focus of brain metastasis and were managed by surgery or radiotherapy for the same.
\end{abstract}

\section{Case history}

A forty-five year old female presented at our institute after her cholecystectomy specimen was found to be positive for poorly differentiated adenocarcinoma. At presentation, she still had pain in right hypochondrium and was febrile. On examination, an unhealthy

Correspondence: hivneg@gmail.com

'Department of Radiotherapy and clinical oncology, Government Medical College, Haldwani, Uttarakhand, India.

Full list of author information is available at the end of the article surgical scar with pus discharge was present in right hypochondrium along the subcostal margin. Mild hepatomegaly, approximately two fingers below the subcostal margin in mid-clavicular line, was palpable. The histopathological examination report of operative specimen showed poorly differentiated adenocarcinoma infiltrating full thickness of gallbladder up to serosa (Figure 1). Patient subsequently underwent an extensive medical evaluation. Her haemoglobin was 8 gram\% and her total leucocyte count was raised to 15,200. Neutrophils were raised to $82 \%$. Platelet count was within normal limits. Her bleeding time, clotting time, serum electrolytes were within normal limits. Her liver function tests including bilirubin levels, renal function tests were all within normal limits. Patient was primarily managed with blood transfusion, antibiotics and supportive treatment. She was subsequently planned for six cycles of 5-fluorouracil, adriamycin and Mitomycin-C based chemotherapy as adjuvant treatment, after completion of antibiotic therapy, when the surgical wound had healed completely. She received four cycles of chemotherapy at three weeks interval. One week after her fourth chemotherapy, the patient presented with right sided hemiparesis. She also complained of two episodes of headache with vomiting. Magnetic resonance imaging of brain was done, which showed 31.8x $25.1 \times 32$ millimetres sized space occupying lesion in left high parietal region with moderate peri-lesional oedema leading to pressure over left lateral ventricle with subtle midline shift towards right side. Another lesion of $14.2 \times 12.1 \times 13.2$ millimetres was present at right fronto-parietal region which was peripheral in location with minimal peri-lesional oedema (Figure 2). Considering the size of lesion and the significant symptoms caused by it, left parietal craniotomy with excision of lesion in left parietal region was done. Histopathology of the specimen showed poorly differentiated metastatic adenocarcinoma (Figure 3). There was significant improvement in her symptoms caused by right 


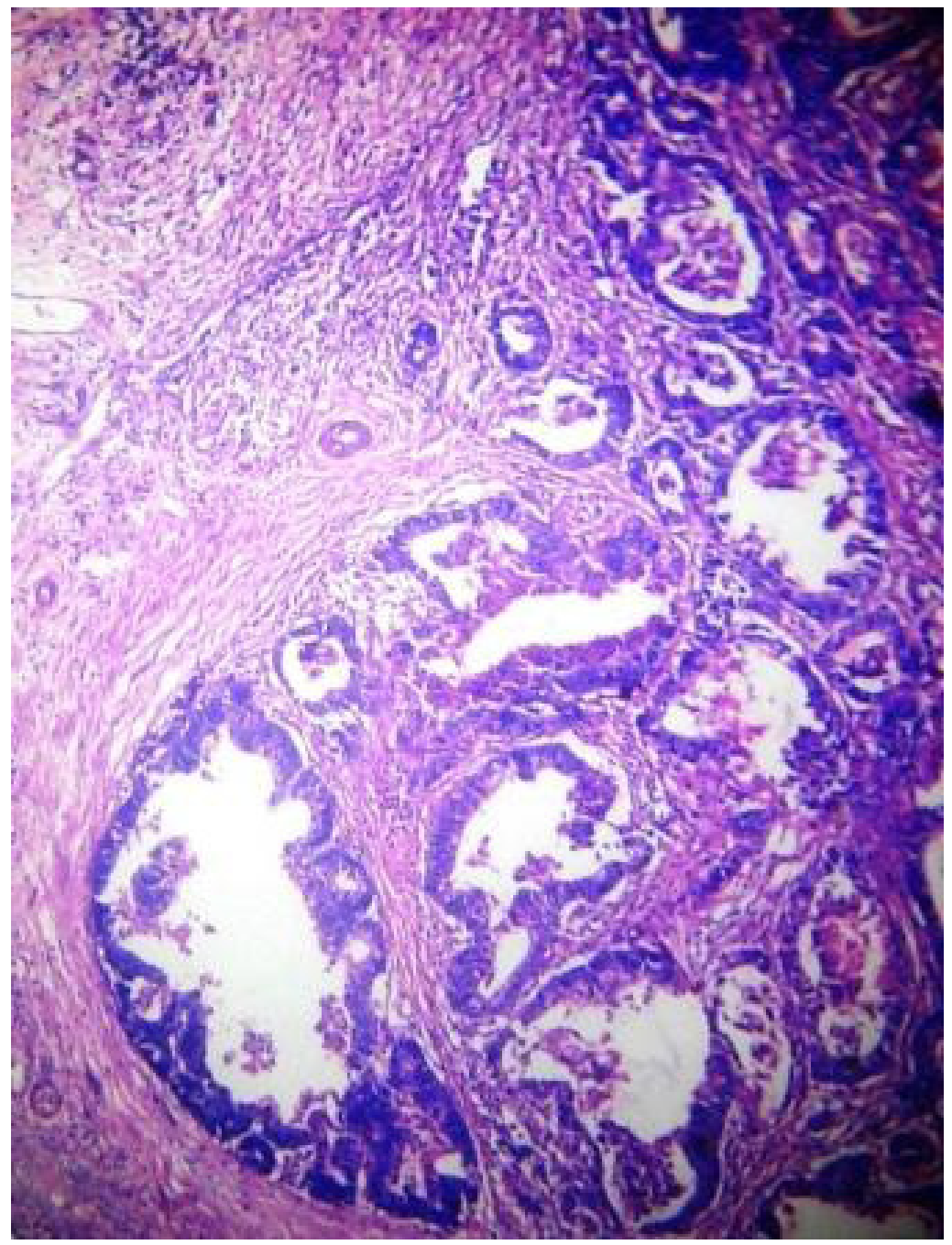

Figure 1: Photomicrograph of histopathology of operative specimen showing poorly differentiated adenocarcinoma infiltrating full thickness of gallbladder up to serosa.

parietal metastatic lesion after the surgery. On complete healing of operative scar, patient was planned for external beam radiotherapy to whole brain to take care of the smaller lesion and other possible micro-metastases. Patient was administered 30 Grays in ten fractions in two weeks' time to whole brain. After her radiation treatment was completed, she was administered two more remaining cycles of chemotherapy at three weeks interval that were initially planned. She tolerated whole treatment well without any complications. PETCT scan, done one month after completion of her chemotherapy, was negative for any active foci in body. Thirteen months after her treatment, she is currently asymptomatic and is doing well.

\section{Discussion}

Systemic metastases from gallbladder carcinoma occur frequently, but involvement of the central nervous system is a rarity. The incidence of central nervous system (CNS) metastases from gallbladder carcinoma is approximately $2 \%$. [1,2] CNS metastases, when present, from a primary gallbladder carcinoma are usually associated with other systemic metastases also. No other metastatic sites were detected in this patient. Patients with brain metastases receiving corticosteroids, surgical, and radiation therapies survive longer than untreated patients. Many authors have reported surgical treatment for single brain metastasis $[3,9,10]$. A randomized trial of patients with systemic cancer 


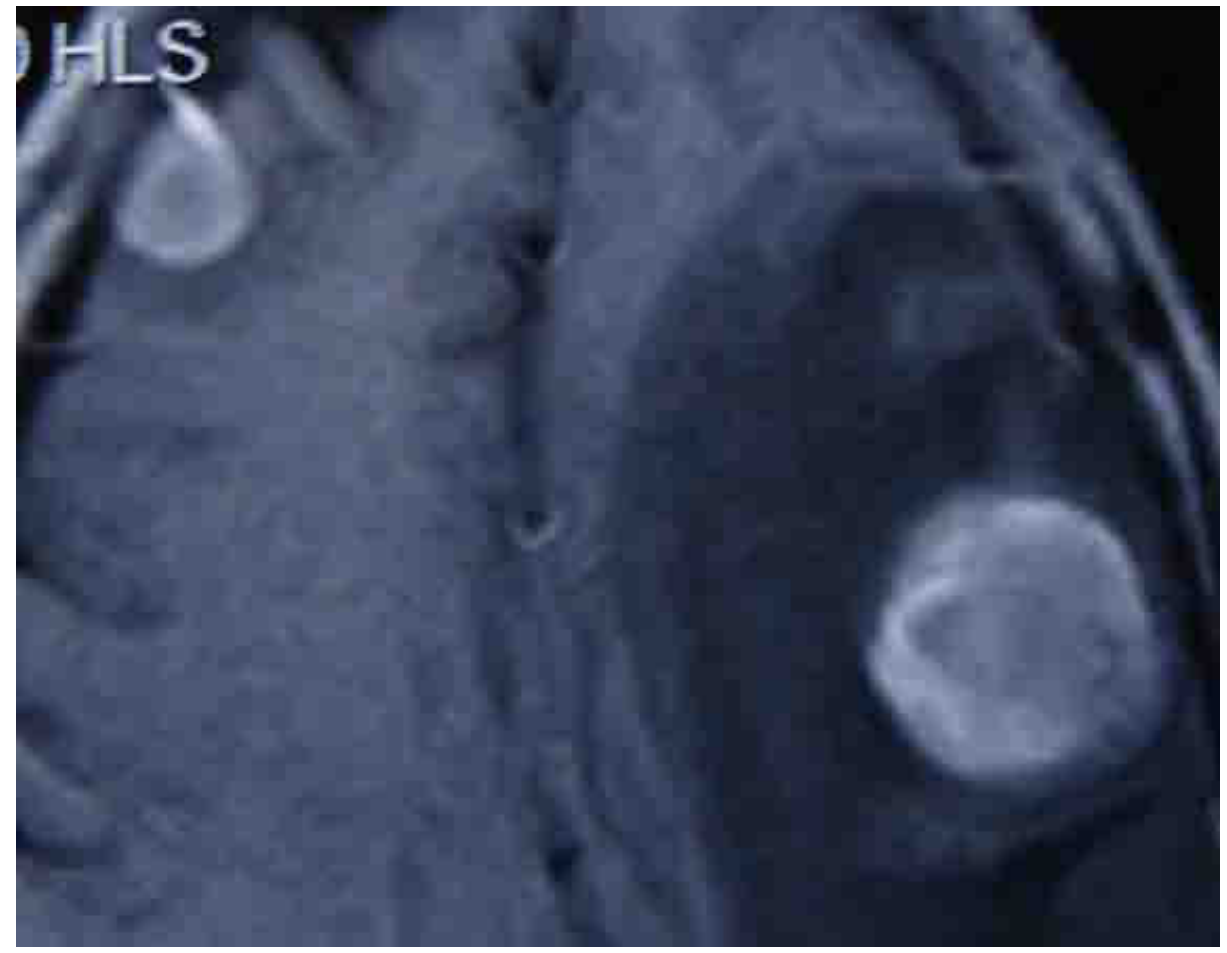

Figure 2: Magnetic resonance imaging showing space occupying lesions in left high parietal region and right fronto-parietal region.

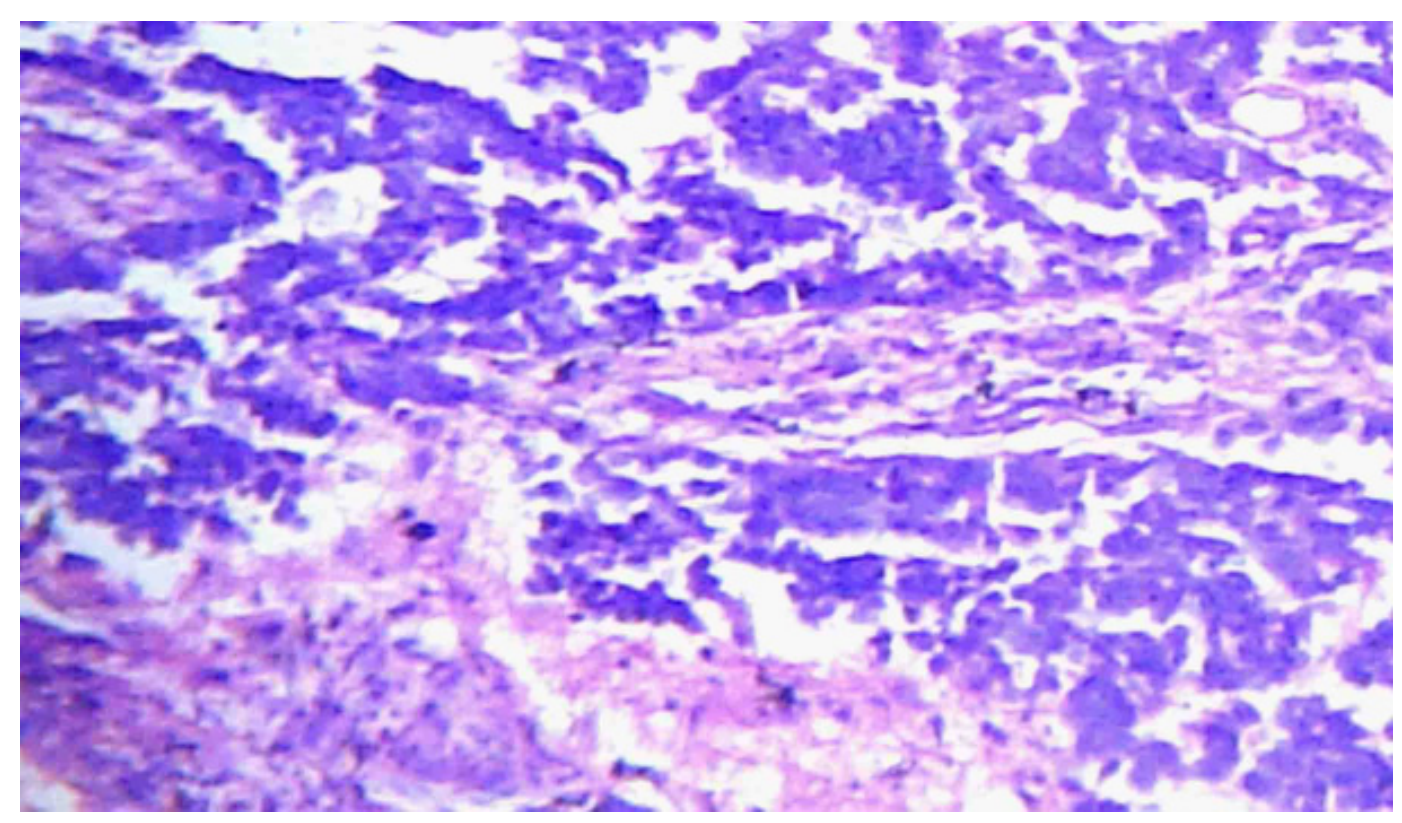

Figure 3: Photomicrograph of histopathology of the operative specimen of brain lesion showing poorly differentiated metastatic adenocarcinoma. 
and a radiological diagnosis of single brain metastasis showed that combined treatment of surgery and adjuvant radiotherapy compared with radiotherapy alone led to a longer survival especially in patients with stable extra-cranial disease. Patients with progressive extracranial cancer had a median overall survival of 5 months irrespective of given treatment [11]. Though brain metastasis from gallbladder carcinoma is very unusual, it should not be overlooked. Clinicians should keep in mind this possibility in patients of gallbladder carcinoma presenting with central nervous system symptoms [8]. There have been reports in past where single lesions in brain were treated by surgical intervention. Such an approach was difficult in this case as both lobes of the brain were involved by two different lesions and patient was not fit for an extensive surgery of the lesions involving both lobes of brain simultaneously or two surgeries at an interval. The residual neurological deficit after surgery for both lesions could have been more than the symptoms patient was having before surgery from one of the lesions while the other metastatic foci was asymptomatic. Thus, surgical excision was done for the larger symptomatic lesion in left parietal region and whole brain radiation therapy was given for treatment of the smaller lesion in the right lobe and other possible micro-metastases followed by combination chemotherapy. Studies have shown benefits of earliest possible introduction of treatment for brain metastases in such cases. Although early treatment has been linked to prolonged survival and improved quality of life, brain metastases represent a late manifestation of $\mathrm{Gl}$ cancers and remain an ominous sign [12].

\section{Conclusion}

Surgery with or without adjuvant radiation therapy has been indicated for solitary, peripherally located brain metastases. In patients with multiple metastases, surgery, radiation therapy or chemotherapy alone or in combination are the options available. The treatment plan in patients with multiple metastases needs to be individualized keeping in mind the number and location of symptomatic metastatic lesions, the possible neurological deficits after surgery, stability and progression of the metastatic lesions; and fitness and tolerability of patient for combination therapy. Such individualized treatment can provide palliation to patients with improvement of quality of life; without adding to treatment related complications.

\footnotetext{
Author details

${ }^{2}$ Department of Radiotherapy and Clinical oncology, V.C.S.G.G.M.S.\&R.I., Srinagar Garhwal, Uttarakhand, India.

${ }^{3}$ Department of Radiotherapy, Delhi state

cancer institute, New Delhi, India.

Authors' contributions

\#These authors contributed equally to this work.

Conflicting Interest

Authors declare that they have no conflicting interests.
}

\section{Article history}

Editor: Hanlin L. Wang, University of Nebraska Medical Center, USA. EIC: G.J.Peters, VU University Medical Center, Netherlands. Received: 25-Dec-2011 Revised: 02-Feb-2012

Accepted: 03-Feb-2012 Published: 20-Mar-2012

\section{References}

1. Sons, H. U. et al. Carcinoma of the gallbladder: autopsy findings in 287 cases and review of the literature. J Surg Oncol 28, 199-206.

2. Vaittinen, E. Carcinoma of the gall-bladder. A study of $\mathbf{3 9 0}$ cases diagnosed in Finland 1953-1967. Ann Chir Gynaecol Fenn Supp/ 168, 1-81.

3. Chan, R. C. et al. Solitary cerebral metastasis: the effect of craniotomy on the quality and the duration of survival. Neurosurgery 11, 254-257.

4. Hirano, A. et al. [Metastatic tumors in the central nervous system. The neuropathological point of view (Part 1) (author's transl)]. No Shinkei Geka 8, 509-518.

5. Paillas JE, Pellet W, Brain metastases, in: Handbook of Clinical Neurology, vol 18. Amsterdam, North- Holland, pp 201-232.

6. Stortebecker, T. P. Metastatic tumors of the brain from a neurosurgical point of view; a follow-up study of 158 cases. J Neurosurg 11, 84-111.

7. Yardeni, D. et al. Neurosurgical management of single brain metastasis. Surg Neurol 21, 377-384.

8. Takano, S. et al. Central nervous system metastasis from gallbladder carcinoma--case report. Neurol Med Chir (Tokyo) 31, 782-786.

9. Gagliardi, F. M. et al. Single metastases in the brain: late results in 325 cases. Acta Neurochir (Wien) 68, 253-262.

10. Galicich, J. H. et al. Surgical treatment of single brain metastasis. Evaluation of results by computerized tomography scanning. J Neurosurg 53, 63-67.

11. Vecht, C. J. et al. Treatment of single brain metastasis: radiotherapy alone or combined with neurosurgery? Ann Neurol 33, 583-590.

12. Go, P. H. et al. Gastrointestinal cancer and brain metastasis: a rare and ominous sign. Cancer 117, 3630-3640. 